\title{
Magnetic properties of stoichiometric and defective $\mathrm{Co}_{9} \mathrm{~S}_{8}$
}

\author{
Marco Fronzi, ${ }^{1,2, *}$ Sherif Abdulkader Tawfik, ${ }^{2}$ Catherine Stampfl, ${ }^{3}$ and Mike Ford ${ }^{2, \dagger}$ \\ ${ }^{1}$ International Research Centre for Renewable Energy, \\ State Key Laboratory of Multiphase Flow in Power Engineering, \\ $X i$ 'an Jiaotong University, Xi'an 710049, Shaanxi, China \\ ${ }^{2}$ School of Mathematical and Physical Science, University of Technology Sydney, Ultimo 2007, New South Wales 2007, Australia \\ ${ }^{3}$ School of Physics, The University of Sydney, Sydney, New South Wales 2006, Australia
}

(Dated: March 13, 2019)

\begin{abstract}
In this paper, we present a detailed study of the stoichiometric and reduced $\mathrm{Co}_{9} \mathrm{~S}_{8}$ pentlandite magnetic properties, based on density functional theory. We analyze both its geometry and electronic properties and show that only by the inclusion of the Hubbard term it is possible to correctly describe $d-d$ splitting, which is necessary to accurately characterize the $\mathrm{Co}_{9} \mathrm{~S}_{8}$ spin configuration and its antiferromagnetic nature. We also analyze the effect of sulfur vacancies and predict the formation of ferromagnetic clusters that give local ferromagnetic character to non-stoichiometric $\mathrm{Co}_{9} \mathrm{~S}_{8}$, which may explain the contradictory experimental results reported in the literature.
\end{abstract}

\section{INTRODUCTION}

Transition metal sulfide (TM-S) nanomaterials have attracted much attention due to their excellent properties and promising applications in electronic, optical, and optoelectronic devices as well as in spintronics. ${ }^{1}$ The production of novel TM-S structures with the desired magnetic properties is crucial for the fabrication of innovative technological devices. ${ }^{1-3}$ In particular, TM-S compounds with antiferromagnetic (AFM) properties are attractive due to the numerous features they offer compared to more common ferromagnetic (FM) compounds. These features include a stable coupled moment when exposed to external magnetic fields, stability in multiple magnetic configurations, and higher magnetic ordering temperatures. Furthermore, AFM characteristics typically appear in insulating materials, with only few examples of AFM metals, which makes the investigation of such systems attractive.

Cobalt sulfide is present in several phases with different stoichiometries. The magnetic susceptibility measurements of various phases of cobalt sulfides reveal that $\mathrm{CoS}$ and $\mathrm{Co}_{4} \mathrm{~S}_{3}$ exhibit temperature-independent paramagnetism, $\mathrm{Co}_{3} \mathrm{~S}_{4}$ has AFM characteristics, and $\mathrm{CoS}_{2}$ is ferromagnetic with a Curie temperature of $116 \mathrm{~K}^{4,5}$ However, observation of a high magnetic-ordering temperature in transition metal sulfides is uncommon. ${ }^{6}$

Metallic $\mathrm{Co}_{9} \mathrm{~S}_{8}$ has been investigated for its catalytic properties, however its magnetic properties have only been partially analyzed, and the literature reports contradictory results. ${ }^{4,6,7}$ In particular, the paper by Heidelberg et al. ${ }^{7}$ which is based on susceptibility measurement, suggests an AFM character occuring below the Néel temperature of $300 \mathrm{~K}$, which was also reported by Lai et al. ${ }^{4}$ On the other hand, Kumar et al. ${ }^{6}$ concluded that $\mathrm{Co}_{9} \mathrm{~S}_{8}$ exhibits the magnetic hysteresis typical of a ferromagnet at room temperature, with a magnetization peak at low temperature, Luo et al. ${ }^{8}$ observed a paramagnetic character in $\mathrm{Co}_{9} \mathrm{~S}_{8}$ microspheres at room temperature, whereas Pasquariello et al. reported an observed a ferromagnetism resulting from the formation of a small amount of cobalt metal during synthesis. ${ }^{9}$ Furthermore, computational-theoretical studies report no magnetic order, and classify this material as non-magnetic. ${ }^{10,11}$

These contradictory results in the literature suggest that the measured magnetic nature of $\mathrm{Co}_{9} \mathrm{~S}_{8}$ may be due to the specific experimental conditions. The local stoichiometry of the crystal, in particular, could play a fundamental role in the measured macroscopic properties, as reported for similar crystals. ${ }^{12-16}$

Here we describe our first-principles investigation, to elucidate the magnetic nature of $\mathrm{Co}_{9} \mathrm{~S}_{8}$. We analyse the geometries, energetics and electronic structure of stoichiometric and defective $\mathrm{Co}_{9} \mathrm{~S}_{8}$, with a focus on the role of sulfur vacancies in the magnetic order of this crystal.

\section{CALCULATION METHODS}

We performed the calculations presented in this work using Density Functional Theory (DFT), within the Generalized Gradient Approximation (GGA) using the Perdew-Burke-Ernzerhof functional (PBE) for the exchange-correlation term. ${ }^{17}$ To describe the $d-d$ splitting in a way that is consistent with experimental results, we must include a Hubbard correction with $U_{\text {eff }}\left(\mathrm{Co}_{3 d}\right)$ between $2 \mathrm{eV}$ and $5 \mathrm{eV}$. Here, we use $U_{\text {eff }}\left(\mathrm{Co}_{3 d}\right)=5 \mathrm{eV}$, in line with Assadi et al., and $U\left(\mathrm{Co}_{3 d}\right)=0 \mathrm{eV}$ to measure the effect of the $d-d$ splitting on the calculated magnetic order. ${ }^{18-20}$. We use periodic boundary conditions and a plane waves basis set as implemented in the simulation package VASP, wherein the core-valence interaction is described by Projector Augmented Wave Pseudopotential. ${ }^{21-24}$ We use a cut-off energy for the valence electrons of $500 \mathrm{eV}$, and use a $3 \times 3 \times 3 \mathrm{k}$-point mesh for the primitive cells consisting of 17 atoms, and for the super-cell consisting of 34 atoms constructed by merging two primitive cell along the $\mathrm{x}$ direction. We performed full geometry optimization including spin polarization, with the convergence criteria for the energy and forces being $10^{-6} \mathrm{eV}$ and $10^{-2} \mathrm{eV} / \AA$, respectively. 


\section{RESULTS}

\section{A. Geometry and Electronic Structure of Stoichiometric $\mathrm{Co}_{9} \mathrm{~S}_{8}$}

$\mathrm{Co}_{9} \mathrm{~S}_{8}$ crystalyzes in a $\mathrm{Fm} \overline{3} \mathrm{~m}$ pentlandite structure with a measured lattice constant of $9.92 \AA \AA^{25,26}$ After geometry optimization, we obtained a small distortion of the primitive cell from the perfect trigonal structurea lattice and a lattice constant of $\mathrm{a}=10.20 \AA$, which is larger than the one reported in other computational studies due to the different magnetic order obtained and the effect of the Hubbard correction, and also larger the experimental value in line with other computational works that adopt a similar methodology. ${ }^{2710,11,28-30}$ Primitive cell of $\mathrm{Co}_{9} \mathrm{~S}_{8}$ pentlandite can be thought of as a packing of four Co atoms bonded to $\mathrm{S}$ atoms in tetrahedral coordination $\left(\mathrm{Co}^{\text {tetra }}\right)$, forming two $\mathrm{Co}_{4} \mathrm{~S}_{4}$ complexes, which are connected through a Co site in a octahedral coordination $\left(\mathrm{Co}^{\text {octa }}\right)$, as schematically depicted in Fig.1. Six coordinated Co atoms have symmetry $\mathrm{O}_{h}$, and four coordinated Co are trigonally distorted with local $\mathrm{C}_{3 v}$ symmetry, with one short and three longer Co-S distances. The two non equivalent sulfur sites (S1 and S2) are either shared by $\mathrm{Co}^{\text {octa }}$ and $\mathrm{Co}^{\text {tetra }}$, with calculated bond lengths for $\mathrm{Co}^{\text {octa }}{ }_{-} \mathrm{S} 1$ of $2.52 \AA$ and for $\mathrm{Co}^{\text {tetra }}{ }_{-} \mathrm{S} 1$ of 2.37 $\AA$, or shared only by $\mathrm{Co}^{\text {tetra }}$ with a $2.33 \AA \mathrm{Co}^{\text {tetra }}$-S2 distance.

We start with an intuitive consideration of the possible magnetic nature of $\mathrm{Co}_{9} \mathrm{~S}_{8}$ within crystal field theory (CFT), while also considering that the formal oxidation states approach does not consider the mixed nature of the atomic bonding in real crystals. CFT predicts the splitting of the $3 d$ electrons of Co, which depends only on atomic coordination. For example, in the more common cobalt oxide $\mathrm{Co}_{3} \mathrm{~S}_{4}$, the $\mathrm{Co}$ (II)/Co(III) ions are considered to occupy the tetrahedral and octahedral sites, respectively. However, the description of the oxidation state of $\mathrm{Co}_{9} \mathrm{~S}_{8}$ may differ from the more common phases, due to the unusually low oxidation state of octahedral Co. The low concentration of S anions, indicates that Co atoms are in a low oxidation state with a mix of $\mathrm{Co}^{\text {tetra }}$ in $d^{7}$, and possibly that the uncommon $\mathrm{Co}^{\text {octa }}$ species are also in low oxidation state $d^{7}$, with a density of $8 / 9$ $\mathrm{Co}^{\text {tetra }}$ and $1 / 9 \mathrm{Co}^{\text {octa }}$. Here, the stability of the $\mathrm{Co}$ in the $3 d^{7} 4 s^{0}$ configuration for fourfold tetrahedral coordinated sites is expected, having been demonstrated in symilar crystals by Walsh et al. ${ }^{18}$

The local crystal field splits the tetrahedral Co $3 d$ states into low energy $\mathrm{e}_{g}$ and high energy $\mathrm{t}_{2 g}$ with a $d-d$ energy split of the order of $1.5-2.0 \mathrm{eV}$, which results in a high spin configuration $\left(\mathrm{e}_{g}^{4} \mathrm{t}_{2 g}^{3}\right)$ with a local magnetic moment $\mu_{B} \sim 3 .^{31}$

On the other hand, octahedral Co splits $3 d$ levels into high energy $\mathrm{e}_{g}$ and low energy $\mathrm{t}_{2 g}$ that, given the $d-d$ energy split, favour a low spin configuration with no magnetic moment for the common $d^{6}$ electron configuration. However, since Co in a low oxidation state, the electronic

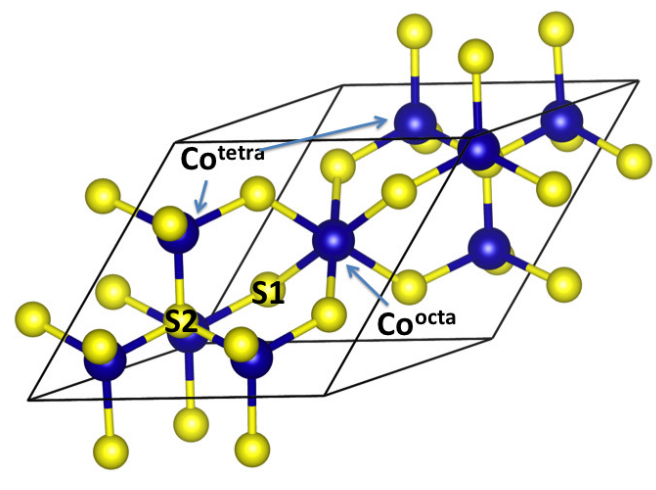

FIG. 1. (Color online) Schematic representation of the $\mathrm{Co}_{9} \mathrm{~S}_{8}$ bulk unit cell employed in our calculations. Blue and yellow spheres represent cobalt and sulfur atoms, respectively.

configuration of this specific crystal, may present a significant deviation from this analysis. Our results consistently show that for each of the energetically favourable and stable structures, tetrahedral Co favours a high spin configuration with a magnetic moment $\sim 2.65 \mu_{B}$. However, the calculated octahedral Co magnetic moment is $\sim 1.68 \mu_{B}$, suggesting that $\mathrm{Co}^{\text {octa }}$ is forced into a $d^{7}$ configuration, as shown in schematic in Fig.2.

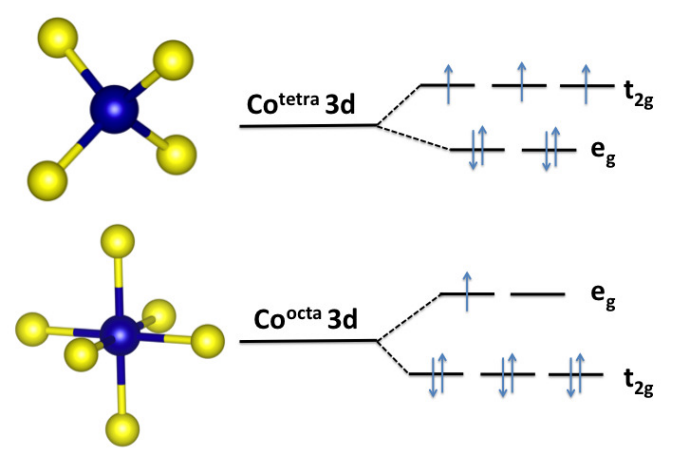

FIG. 2. (Color online) Schematic representation of $d-d$ splitting, as described within Crystal Field Theory, for $\mathrm{Co}_{9} \mathrm{~S}_{8}$ octahedral and tetrahedral Co in the $d^{7}$ configuration.

The calculated energies for the unit cell in different spin configuration, i.e. FM and three AFM, favour an AFM character, with an energy gain of $\Delta_{E_{A F M}-E_{F M}}=0.4 \mathrm{eV}$ per unit formula, as reported in Tab.3. Figure 3 shows the favourable spin configuration AFM2, together with the other configurations used in our calculations. The magnetic moment at $\mathrm{Co}^{\text {tetra }}$ is $2.65 \mu_{B}$ and that at $\mathrm{Co}^{\text {octa }}$ is $1.68 \mu_{B}$, which gives a good description of the predicted spin configuration of $\mathrm{Co}$ (II) under different coordination. Also, the Bader analysis confirms the oxidation state predicted by the $d^{7}$ configuration for both the Co sites (see Fig. 2), predicting a total charge of 8.04 valence electrons for Co ${ }^{\text {tetra }}$ with $\sim 7.02$ electrons in $d$ states, and 8.35 valence electrons for $\mathrm{Co}^{\text {octa }}$ with $\sim 7.20$ electrons in $d$ states. 
TABLE I. Energy per formula unit and magnetic moment at Co sites of non-magnetic (NM), ferromomagnetic (FM) and antiferromagnetic (AFM) configurations, calculated for $U=5$ $\mathrm{eV}$.

\begin{tabular}{llll}
\hline Conf & Energy $(\mathrm{eV})$ & $\mathrm{Co}^{\text {octa }}\left(\mu_{B}\right)$ & $\mathrm{Co}^{\text {tetra }}\left(\mu_{B}\right)$ \\
\hline NM & -65.98 & - & - \\
FM & -80.51 & 1.69 & 2.65 \\
AFM1 & -80.91 & 1.80 & 2.61 \\
AFM2 & -80.92 & 1.68 & 2.65 \\
AFM3 & -80.89 & 1.63 & 2.58 \\
\hline
\end{tabular}

Figure 4 shows the calculated Density of States (DOS), which reveals the AFM nature of the crystal with a metallic character, as indicated by the occupied states at the Fermi level for both spin up and spin down. The projected DOS shows that, in stoichiometric AFM, Co ${ }^{\text {tetra }}$ $3 d$ states are split into the relatively sharp peaks of $\mathrm{e}_{g}$ states (green area in Fig.4b) at $-6.5 \mathrm{eV}$, which then broaden into $t_{2 g}$ states (red area in Fig.4b) at $\sim-4 \mathrm{eV}$, with a $d-d$ distance of $\sim 2 \mathrm{eV}$, which is in accordance with experimental value. However, $\mathrm{t}_{2 g}$ seems to partially remove the degeneracy, due to the local distortion of the lattice at the $\mathrm{Co}^{\text {tetra }}$ which alters the local $\mathrm{T}_{d}$ symmetry to $\mathrm{C}_{3 v}$. The narrow $\mathrm{Co}^{\text {octa }} 3 d \mathrm{e}_{g}$ states are centered below the Fermi energy, at about $-1.5 \mathrm{eV}$, whereas the $\mathrm{t}_{2 g}$ has a peak at $-4.5 \mathrm{eV}$, with additional peaks due to hybridization via sulfur with the tetrahedral Co $3 d$ states at $-6.5 \mathrm{eV}$. The calculated $d-d$ splitting of $\sim 3 \mathrm{eV}$ is consistent with the predicted orbital configuration of $\mathrm{Co}$ in octahedral coordination.

Burdett et al. and Chauke at al. state that at low energies, below the Fermi energy, the Co $3 d$ and S $3 p$ occupied states suggest a bonding interaction. At higher energies, $\mathrm{S} p$-states are present, however this range has a predominance of Co $3 d$ states with sharp peaks, which suggests a non-bonding character. Above the Fermi energy, the character of S $3 p$ and Co $3 d$ states suggest an anti-bonding character, which indicates that the Fermi level lies between the non-bonding and anti-bonding states. $^{32,33}$ To analyse the bonding nature of Co-S, we determined the crystal overlap hamiltonian population (COHP) by multiplying the DOS by the corresponding element of the Hamiltonian, which is conventionally considered to have opposite sign, and yields additional information regarding the bonding character: positive and negative values indicate bonding and antibonding interactions, respectively. Our COHP analysis, suggests that there is a bonding-antibonding transition at $\sim-4 \mathrm{eV}$ for $\mathrm{Co}^{\text {tetra }}$ with a non-bonding character around the Fermi level. On the other hand, for $\mathrm{Co}^{\text {octa }}$ the same transition occurs at $\sim-2 \mathrm{eV}$, with non-bonding states only above the Fermi level, indicating an anti-bonding character for $\mathrm{Co}^{\text {octa }} \mathrm{e}_{g}$, which is consistent with theoretical considerations. The antibonding states at high energies suggest an electronic instability, that reflects, in the electronic reconfiguration, a vacancy formation that breaks the lat-
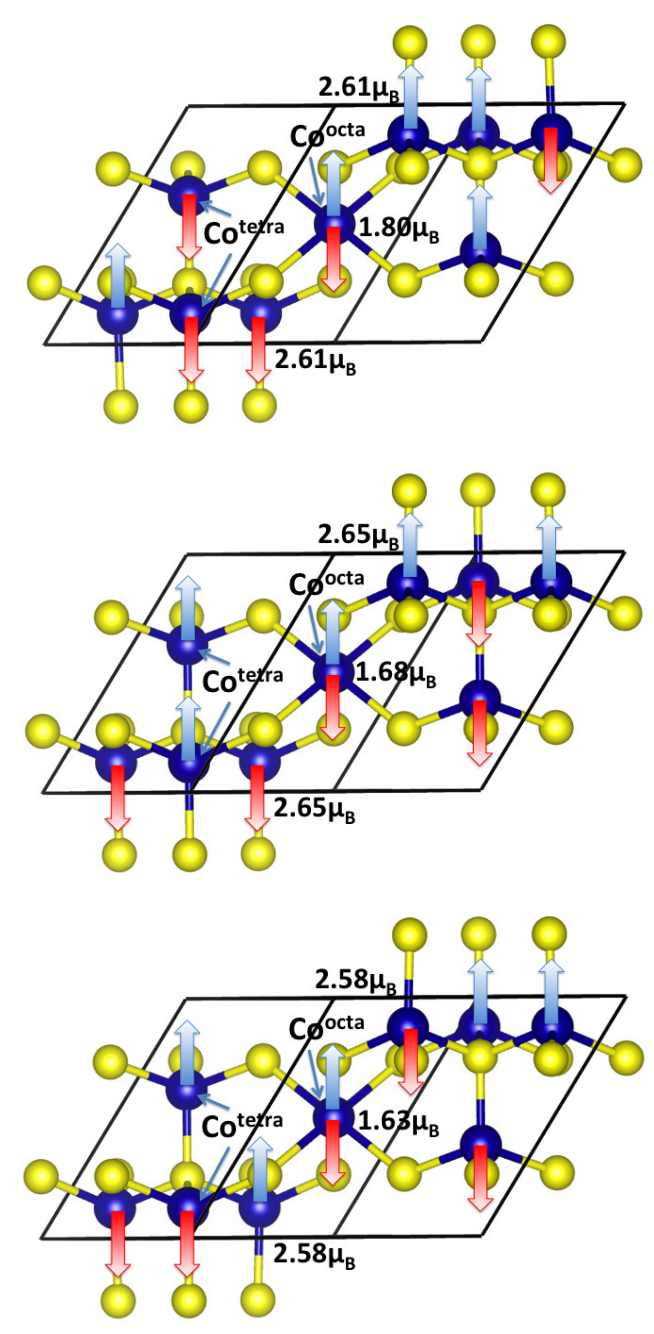

FIG. 3. (Color online) Schematic representation of three different AFM configurations in stoichiometric $\mathrm{Co}_{9} \mathrm{~S}_{8}$. From top to bottom they correspond to AFM1 AFM2 and AFM3 in Tab.I. The blue and red arrows indicate spin-up and spindown. The double arrow at $\mathrm{Co}^{\text {octa }}$ indicate alternating spinup and spin-down in adjacent cells used in large cell calculations.

tice symmetry.

To test the validity of our choice for the Hubbard term, we also repeated the calculations with different values of $U$. For a Hubbard correction $U=0 \mathrm{eV}$, the results do not consistently describe a spin configuration with CFT considerations, instead favouring a non magnetic electron configuration. This is because DFT wrongly describes the $d$ orbital energetics, with no $d-d$ splitting, as shown in Fig.5(a), where the overlapping $d$ orbitals cause a net nil magnetic moment. The same effect is obtained for values of $U$ up to $1 \mathrm{eV}$. When $U$ increases from $1.5 \mathrm{eV}$ to $7 \mathrm{eV}$ a net spin at $\mathrm{Co}^{\text {tetra }}$ sites is obtained, with local magnetic moment $0.70 \leq \mu_{B} \leq 2.70$, favouring an AFM configuration. Since the crystal stoichiometry is close to that of CoS, and with a high density of 

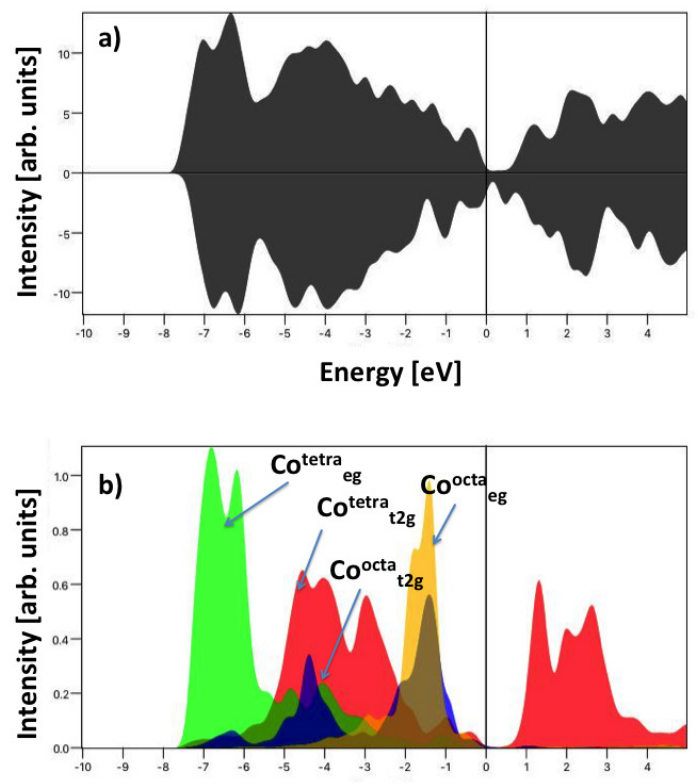

Energy $[\mathrm{eV}]$

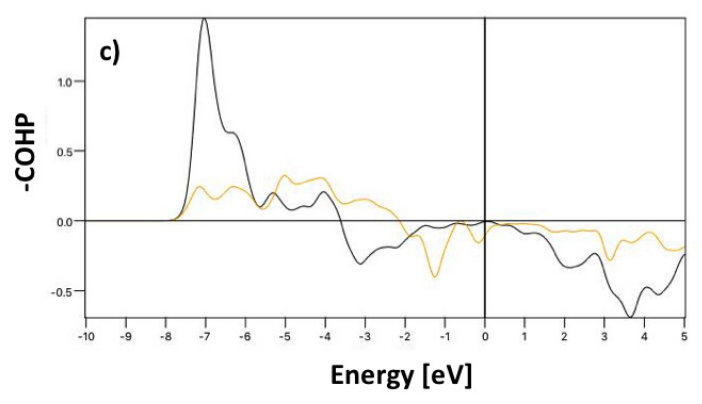

FIG. 4. (Color online) a) Total Density of States, b) projected Density of States of the Co ${ }^{\text {octa }}$ (blue and yellow) and Co tetra $^{\text {trat }}$ (red and green), c) Crystal Overlap Hamiltonian Population of the $\mathrm{Co}^{\text {octa }}-\mathrm{S}$ (yellow line) $\mathrm{Co}^{\text {tetra }}-\mathrm{S}$ (black line) bonds in stoichiometric $\mathrm{Co}_{9} \mathrm{~S}_{8}$, corresponding to the AFM2 configuration in Tab.I.

Co sites in tetrahedral coordination, we expect from basic consideration to have an electronic configuration that is close to that of tetrahedral $\mathrm{Co}(\mathrm{II})$, with a theoretical spin $\mu_{B}=\sqrt[2]{4 S(S+1)} \approx 3.87$, and with a total magnetization $\mu_{B}=\sqrt[2]{4 S(S+1)+L(L+1)} \approx 4.58$. This value measured in similar structures is $\mu_{B} \sim 4.20$, which confirms that the spin at $\mathrm{Co}^{\text {tetra }}$ is close to the theoretical one. The presence of $\mathrm{Co}^{\text {octa }}$, will generate a deviation from this scenario, which however should be relatively small due to the low density of octahedral sites. We obtain a spin configuration close to the ideal tetrahedral $\mathrm{Co}(\mathrm{II})$ with values of $U$ equal or above $5 \mathrm{eV}$, as shown in Fig.5(b). However, value of $U$ higher than $5 \mathrm{eV}$ will result in a non nil a bang gap for spin up that indicate a half metallic character, which is not in line with experimental observations (see Figs.5(c)). ${ }^{6}$

Assuming the second-nearest model, within mean-field
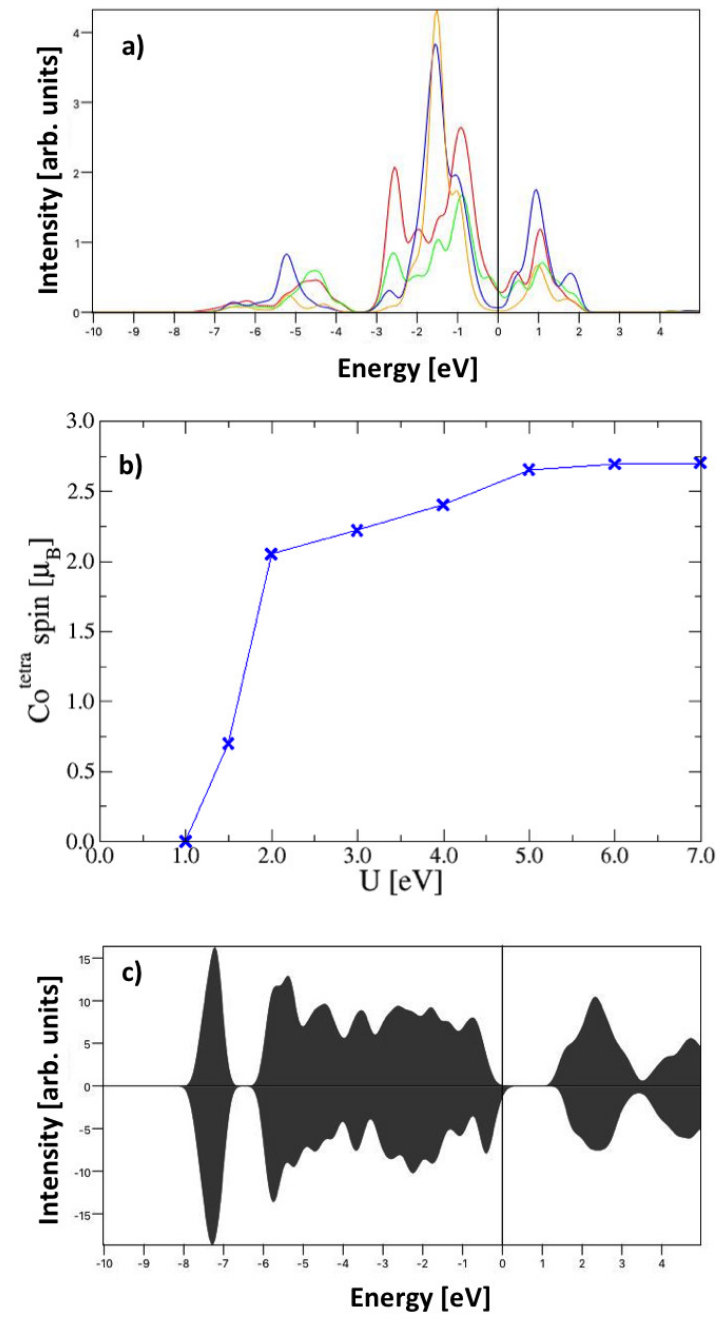

FIG. 5. (Color online) a) projected Density of States of the $\mathrm{Co}^{\text {octa }}$ (blue $\mathrm{t}_{2 g}$ and yellow $\mathrm{e}_{g}$ ) and $\mathrm{Co}^{\text {tetra }}$ (red $\mathrm{t}_{2 g}$ and green $\mathrm{e}_{g}$ ) calculated using the Hubbard correction $U=0$. b) Co $\mathrm{Cotra}^{\text {tet }}$ magnetic moment as a function of the Hubbard correction. c) Total Density of States of stoichiometric $\mathrm{Co}_{9} \mathrm{~S}_{8}$ calculated with $U=6 \mathrm{eV}$.

theory, we can calculate the Néel Temperature $\left(T_{n}\right)$ as follows:

$$
T_{n}=\frac{S(S+1)}{3 k_{B}} \sum_{l=1,2} J_{l},
$$

where $S$ is the total spin, $k_{B}$ is the Boltzmann's constant, and $J_{l}$ is the exchange coupling that runs over the nearest- and next-nearest-neighbors. $J_{1}$ and $J_{2}$ can be extrapolated from energies reported in Tab.I. ${ }^{34-36}$

We can write each AFM energy as a sum of a contribution to the total energy with respect to the FM con- 
TABLE II. Number of nearest-neighbours $(n)$ and nextnearest-neighbours $(m)$ for stoichiometric AFM configurations, in antiferromagnetic coupling with respect to FM.

\begin{tabular}{lll}
\hline Conf & $n$ & $m$ \\
\hline AFM1 & 2 & 0 \\
AFM2 & 2 & 2 \\
AFM3 & 1 & 3 \\
\hline
\end{tabular}

figuration $\left(E_{0}\right)$, and a term that include the exchange integrals with respect to the $n_{i}$ nearest-neighbour and the $m_{i}$ next-nearest-neighbour (Tab.II), as follows:

$$
A F M_{i}=E_{0}+S(S+1)\left[-n_{i} J_{1}-m_{i} J_{2}\right] .
$$

Our calculations give values of $J_{1}=35 \mathrm{meV}$ and $J_{2}=5$ meV, and predict $T_{n}=116 \mathrm{~K}$. However, the estimated $T_{n}$ value strongly depends on the value of the Coulomb parameter. ${ }^{37}$

\section{B. Geometry and Electronic Structure of Defective $\mathrm{Co}_{9} \mathrm{~S}_{8}$}

Two non equivalent sulfur vacancies can form in the super-cell, $\mathrm{V}_{S 1}$ and $\mathrm{V}_{S 2}$, due to fourfold and fivefold coordinated sites, respectively, where S1 forms bonds with $\mathrm{Co}^{\text {octa }}$ and Co ${ }^{\text {tetra }}$, whereas S2 forms bonds only with $\mathrm{Co}^{\text {tetra }}$ (see Fig.1). We calculated the vacancy formation energy to be $6.69 \mathrm{eV}$ for $\mathrm{V}_{S 1}$ and $6.64 \mathrm{eV}$ for $\mathrm{V}_{S 2}$, which indicates that, below $T_{n}$ and in thermodynamic conditions, the vacancy density is extremely low. However, vacancy density may also be affected by the specific synthesis process, and could therefore be high enough to influence the crystal properties. Upon $\mathrm{V}_{S 1}$ formation, the broken symmetry leaves Co $\mathrm{Co}^{\text {octa }}$ in a square pyramidal configuration, removing the $\mathrm{e}_{g}$ degeneration and splitting the $t_{2 g}$ into two levels. Yet, due to the $d^{7}$ electron configuration, no significant change occurs in the magnetic moment of $\mathrm{Co}^{\text {octa }}$. The four $\mathrm{Co}^{\text {tetra }}$ atoms neighbouring $\mathrm{V}_{S 1}$, are arranged in a square planar geometry, with a bond length $\mathrm{Co}^{\text {tetra }}-\mathrm{S}$ of $2.40 \AA$ A. Our calculations point to a change in the local magnetic order, with a favourable FM configuration, as shown in Fig. 6 and reported in Tab.III.

Our pDOS analysis results indicate the magnetic character of the Co cluster (see Fig.7). Co ${ }^{\text {tetra }}$ d peaks with a shift in energy for spin up and down of $1 \mathrm{eV}$ and 2 $\mathrm{eV}$, respectively. Our COHP analysis results suggest the formation of Co-Co bonding states, that together with occupation at the Fermi level, confirm the formation of a Co cluster with FM character.

On the other hand, upon $\mathrm{V}_{S 2}$ formation, $\mathrm{Co}^{\text {tetra }}{ }_{-} \mathrm{S}$ bond lengths at the vacancy site are $2.39 \AA$ and $2.43 \AA$, which breaks the local $\mathrm{T}_{d}$ symmetry of the crystal. The local magnetic moment, shown in Fig.6 and reported in
TABLE III. Energy per formula unit and magnetic moment at Co sites for ferromomagnetic (FM) and antiferromagnetic (AFM) configurations upon vacancy formation, calculated for $U=5 \mathrm{eV}$.

\begin{tabular}{llll}
\hline Conf & Energy $(\mathrm{eV})$ & $\mathrm{Co}^{\text {octa }}\left(\mu_{\mathrm{B}}\right)$ & $\mathrm{Co}^{\text {tetra }}\left(\mu_{\mathrm{B}}\right)$ \\
\hline VS1 FM1 & -73.21 & 1.65 & 2.65 \\
VS1 AFM1 & -73.06 & 0.97 & $1.90 / 2.63$ \\
VS1 AFM2 & -73.05 & 1.04 & $1.96 / 2.60$ \\
\hline VS2 FM2 & -73.30 & 0.90 & $1.90 / 2.65$ \\
VS2 AFM1 & -73.20 & 0.91 & $1.92 / 2.61$ \\
VS2 AFM2 & -72.26 & 1.10 & $1.80 / 2.60$ \\
\hline
\end{tabular}
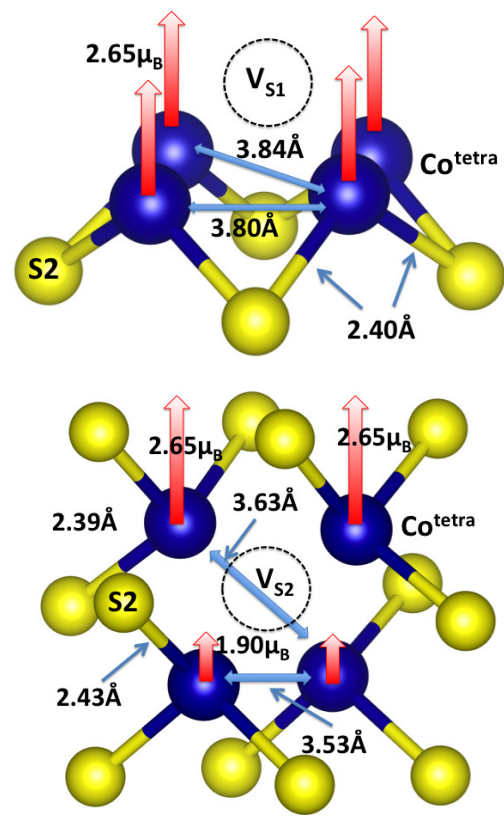

FIG. 6. (Color online) Schematic representation of local FM configurations upon the formation of a sulfur vacancy $\left(\mathrm{V}_{S 1}\right.$ and $\mathrm{V}_{S 2}$ ), which correspond to FM1 (top) and FM2 (bottom) of Tab.III. Bond distances and magnetic moments at each site are indicated. Blue and yellow spheres represent cobalt and sulfur atoms, respectively .

Tab.III, indicates the crystal's local ferromagnetic character, with magnetic moments of 1.90 and $2.65 \mu_{B}$, which suggests a charge transfer, and which is confirmed by the $\mathrm{Co}^{\text {octa }}$ magnetic moment change to $0.90 \mu_{B}$. Our pDOS analysis results confirm the FM order of the Co cluster, with a $\mathrm{Co}^{\text {tetra }}$ and $\mathrm{Co}^{\text {octa }} \mathrm{d}$ peaks distance of $\sim 3$ $\mathrm{eV}$ for both spin-up and spin-down (see Fig.8). However, the COHP analysis results suggest the formation of Co-Co bonding and anti-bonding states in the range $-3 \mathrm{eV}$ to $0 \mathrm{eV}$. The antibonding states close to Fermi level, indicates an internal stress in the lattice, which is in accordance with the calculated distorted local geometry. Also, the magnetic moment of the stable $\mathrm{V}_{S 2}$ configuration FM2, showing that $\mathrm{Co}^{\text {octa }}$ loses a fraction of electron in favour of $\mathrm{Co}^{\text {tetra }}$, confirmed by the Bader 

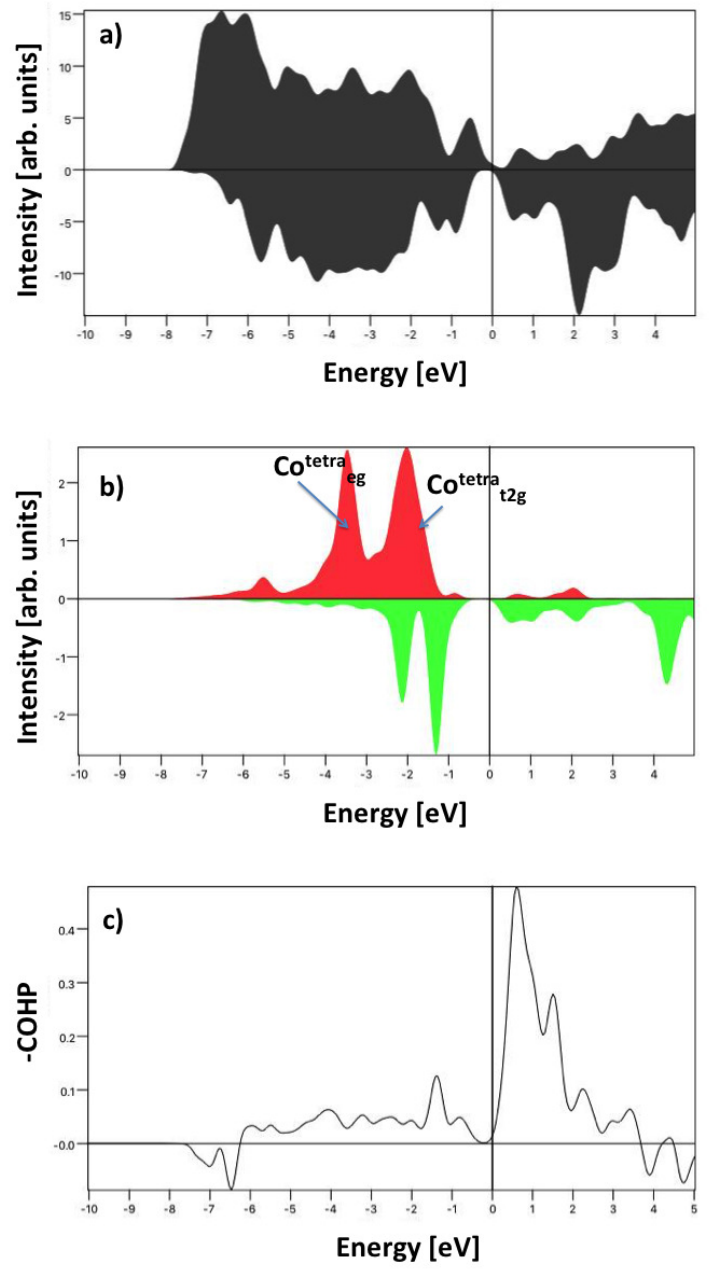

FIG. 7. (Color online) a) Total Density of States, b) projected Density of States of the Co ${ }^{\text {tetra }}$ (red and green), c) Crystal Overlap Hamiltonian Population of the Co ${ }^{\text {tetra }}-\mathrm{Co}^{\text {tetra }}$ bond in non stoichiometric $\mathrm{Co}_{9} \mathrm{~S}_{8}$, corresponding to the FM1 configuration in Tab.III.

analysis that shows an electron transfer of 0.6 electrons, with the orbitals alignment consistent with theoretical considerations. The electron transfer results in a lower magnetic moment for both species, since $\mathrm{e}_{g}$ occupation in $\mathrm{Co}^{\text {octa }}$ decreases and the half full $\mathrm{t}_{2 g}$ states in Co ${ }^{\text {tetra }}$ increases, with a resulting reduction of the total spin for both configurations. The results regarding the magnetic order of non-stoichiometric $\mathrm{Co}_{9} \mathrm{~S}_{8}$ show how this crystal could in principle have either AFM or ferrimagnetic (FiM) character, depending on the specific stoichiometry and density of sulfur vacancies. However, although we calculated a very low vacancy concentration in thermodynamic conditions, the synthesis technique may significantly affect the stoichiometry, which may explain the different measurements reported in the literature.
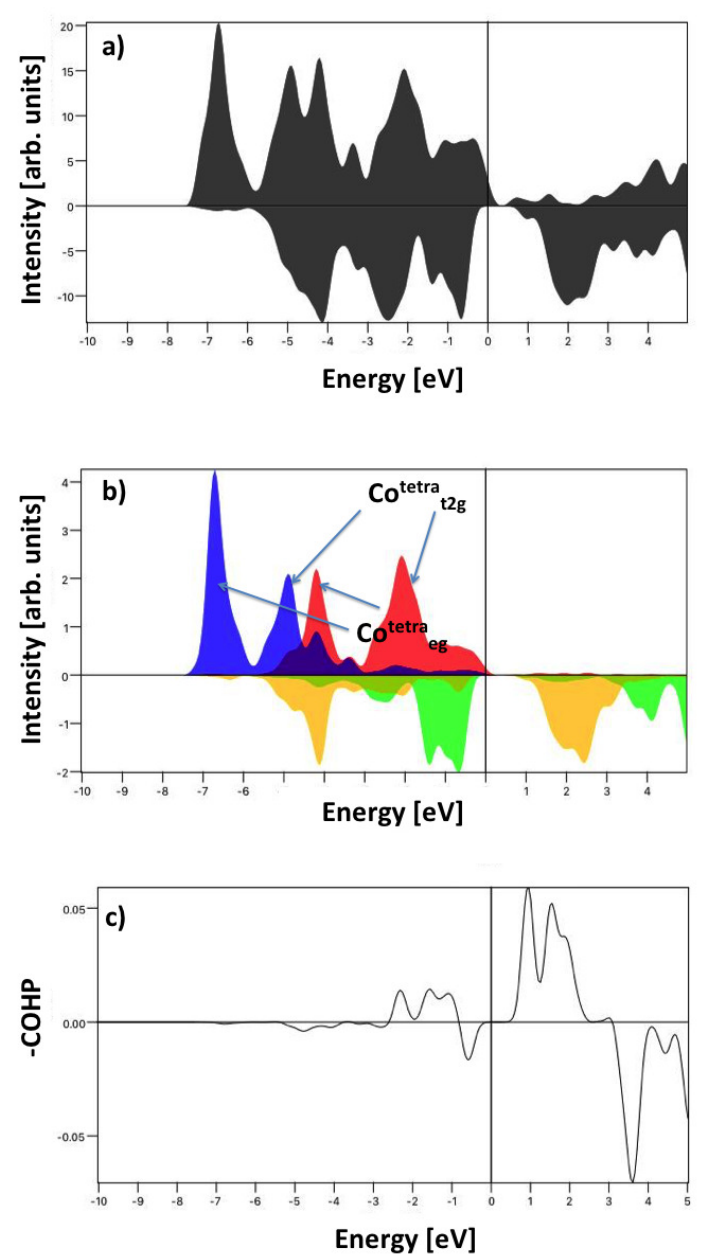

FIG. 8. (Color online) a) Total Density of States, b) projected Density of States of the Co Cotra $^{\text {ted and green), c) Crystal }}$ Overlap Hamiltonian Population of the $\mathrm{Co}^{\text {tetra }}-\mathrm{Co}^{\text {tetra }}$ bond in non stoichiometric $\mathrm{Co}_{9} \mathrm{~S}_{8}$, corresponding to the FM2 configuration in Tab.III.

\section{CONCLUSIONS}

In this paper we analysed the geometry and electronic structure of stoichiometric and reduced $\mathrm{Co}_{9} \mathrm{~S}_{8}$ pentlandite. We compared the results using DFT and $\mathrm{DFT}+U$, and show that DFT incorrectly describe the $d-d$ orbital splitting, which is an essential factor in the correct prediction of the magnetic order of $\mathrm{Co}_{9} \mathrm{~S}_{8}$. We calculated a favourable AFM order with a metallic character for the stoichiometric form with a gain of $\sim 0.4$ $\mathrm{eV}$ as compared to the FM configuration, we estimate exchange parameters and predict the Neél temperature to be $116 \mathrm{~K}$. However, in non-stoichiometric $\mathrm{Co}_{9} \mathrm{~S}_{8}$, we predicted the formation of Co FM clusters, which may explain the contradictory experimental results reported in the literature. 


\section{ACKNOWLEDGEMENTS}

The authors gratefully acknowledge the financial support of the National Natural Science Foundation of China (No. 51323011), and the Australian Government through the Australian Research Council (ARC DP16010130). The theoretical calculations in this research were under- taken with the assistance of resources from the National Computational Infrastructure (NCI), which is supported by the Australian Government. The theoretical calculations in this work were also supported by resources provided by the Pawsey Supercomputing Centre with funding from the Australian Government and the Government of Western Australia.
* Corresponding author

E-mail: Marco.Fronzi@uts.edu.au

$\dagger$ Corresponding author

E-mail: Mike.Ford@uts.edu.au

1 S. Wolf, D. Awschalom, R. Buhrman, J. Daughton, S. Von Molnar, M. Roukes, A. Y. Chtchelkanova and D. M. Treger, Science, 2001, 294, 1488-1495.

2 C. I. Pearce, R. A. D. Pattrick and D. J. Vaughan, Reviews in Mineralogy and Geochemistry, 2006, 61, 127-180.

3 N. Zibouche, A. Kuc, J. Musfeldt and T. Heine, Annalen der Physik, 2014, 526, 395-401.

4 C.-H. Lai, M.-Y. Lu and L.-J. Chen, J. Mater. Chem., 2012, 22, 19.

5 S. Miyazaki, Satoshi ans Masafumi and N. Suzuki, Journal of Magnetism and Magnetic Materials, 1998, 177, 13671368.

6 N. Kumar, N. Raman and A. Sundaresan, Z. Anorg. Allg. Chem., 2014, 640, 1069.

7 R. F. Heidelberg, A. H. Luxem, S. Talhouk and J. J. Banewicz, Inorg. Chem., 1996, 5, 194.

8 J. Luo and Y. Cui, Nanoscience and Nanotechnology, 2016, 16(4), 4164-4168.

9 D. M., Pasquariello, R. Kershaw, J. D. Passaretti, K. Dwight, and A. Wold, Inorganic Chemistry, Vol. 23, No. 7, 1984, 1994, 23, 872.

10 Materials Project, http://www.materialsproject.org.

11 A. Jain, S. P. Ong, G. Hautier, W. Chen, W. D. Richards, S. Dacek, S. Cholia, D. Gunter, D. Skinner, G. Ceder and K. Persson, APL Materials, 2013, 1, 011002.

12 F. Wang, J. Kim, G. D. Gu, Y. Lee, S. Bae and Y.-J. Kim, J. App. Phys., 2013, 113, 063909.

13 A. D. Christianson, M. D. Lumsden, M. Angst, Z. Yamani, W. Tian, R. Jin, E. A. Payzant, S. E. Nagler, B. C. Sales and D. Mandrus, Phys. Rev. Lett., 2008, 100, 107601.

14 J. Bourgeois, M. Hervieu, M. Poienar, A. M. Abakumov, E. Elkaïm, M. T. Sougrati, F. Porcher, F. Damay, J. Rouquette, G. Van Tendeloo, A. Maignan, J. Haines and C. Martin, Phys. Rev. B, 2012, 85, 064102.

15 J. de Groot, K. Marty, M. D. Lumsden, A. D. Christianson, S. E. Nagler, S. Adiga, W. J. H. Borghols, K. Schmalzl, Z. Yamani, S. R. Bland, R. de Souza, U. Staub, W. Schweika, Y. Su and M. Angst, Phys. Rev. Lett., 2012, 108, 037206.

16 J. Mazo-Zuluaga, J. Restrepo and J. Mejía-López, Journal of Physics: Condensed Matter, 2008, 20, 195213.
17 J. P. Perdew, K. Burke and M. Ernzerhof, Phys. Rev. Lett., 1996, 77, 3865.

18 A. Walsh, S.-H. Wei, Y. Yan, M. M. Al-Jassim, J. A. Turner, M. Woodhouse and B. A. Parkinson, Phys. Rev. $B, 2007, \mathbf{7 6}, 165119$.

19 W. L. Roth, J. Phys. Chem. Solids, 1964, 25(1), 1.

20 M. H. N. Assadi and H. Katayama-Yoshida, Journal of Physics: Condensed Matter, 2015, 27, 175504.

21 G. Kresse and J. Hafner, J. Phys.: Condens. Matt., 1994, 6, 8245 .

22 G. Kresse and D. Joubert, Phys. Rev. B, 1999, 59, 1758.

23 P. Raybaud, G. Kresse, J. Hafner and H. Toulhoat, J. Phys.: Condens. Matter, 1997, 9, 11085?11106.

24 D. R. Hamann, M. Schl'uter and C. Chiang, Phys. Rev. Lett., 1979, 43, 1494.

25 R. W. G. Wyckoff, Crystal Structures, John Wiley \& Sons, NewYork, 1964, p. 222.

26 S. Geller, Aceta Cryst., 1962, 15, 1195.

27 R. Ranjan, H. S. Nabi and R. Pentcheva, Journal of Physics: Condensed Matter, 2007, 19, 406217.

${ }^{28}$ G. Bergerhoff, R. Hundt, R. Sievers and I. Brown, Journal of Chemical Information and Computer Sciences, 1983, 23, 66-69.

29 F. Karlsruhe, Inorganic Crystal Structure Database, http: //icsd.fiz-karlsruhe.de.

30 M. E. Arroyo-de Dompablo, A. Morales-Garcia and M. Taravillo, J. Chem. Phys., 2011, 135, 054503.

31 H. A. Weakliem, J. Chem. Phys., 1962, 36, 2117.

32 J. K. Burdett and G. J. Miller, J. Am. Chem. Soc., 1987, 109, 4081-4091.

${ }^{33}$ H. R. Chauke, D. Nguyen-Manh, P. E. Ngoepe, D. G. Pettifor and S. G. Fries, Phys. Rev. B: Condens. Matter, 2002, 66, 155105 .

34 J. Lee, N. Sai and A. A. Demkov, Physical Review B, 2010, 82, 235305.

35 P. Larson and W. R. Lambrecht, Journal of Physics: Condensed Matter, 2006, 18, 11333.

36 N. J. C. Ingle and I. S. Elfimov, Physical Review B, 2008, 77, 121202 .

37 S. Shi, A. L. Wysocki and K. D. Belashchenko, Phys. Rev $B, 2009, \mathbf{7 9}, 104404$. 